\title{
IR HEAT FLUXES MEASUREMENTS IN HYPERSONIC PLASMA FLOW
}

Gennaro Cardone*, Antonio Del Vecchio+, Giovanni Maria Carlomagno*

*University of Naples"Federico II", DETEC Department, p.le Tecchio, 80127 Naples (Italy)

+CIRA - Italian Center for Aersopace Research - Via Maiorise, 81043 Capua

(Italy)

The most critical area of the reentry vehicle design is evaluation and the measurement, inside any ground facility, of the aerothermodynamic heating (and catalicity) of the material under test. The study of the aerothermodynamic properties in an hypersonic plasma flow represents one of the most important application fields of the optical diagnostic methodologies. The principal feature of the physical process is the nonequilibrium aspect of the plasma constituents. This peculiarity requires a specified development (theoretical and experimental) of the diagnostic techniques in order to take into account the non-equilibrium effects and the different catalicity of the constituent materials. In this paper, we present the preliminary results of the application of the thermography to the Plasma Wind Tunnel Facility named SCIROCCO, located at CIRA, (nozzle exit diameter of $2 \mathrm{~m}$, maximum overall size of the test article of about $80 \mathrm{~cm}$ ) devoted to test TPS materials of reentry space vehicles in hypersonic high enthalpy flow conditions. A first step to evaluate the applicability of IR thermography to an hypersonic large scale arcjet facility, is the analysis and the evaluation of the radiation emitted and absorbed by the plasma flow impacting the test sample which represent the medium between the IR Thermograph system (detector and window) and the test sample surface. By using ARCSIZ by Aerotherm Co. and H2NS by CIRA codes, we carried out the estimation of all chemical species and relative concentration for the entire set of flow conditions defining the operative map of Scirocco Plasma Wind Tunnel facility. Then, we assume worthy of consideration any chemical species which results to be present with a significant value of concentration in terms of molar fractions. In table 1, the chemical species, present in not negligible amount at the least in one functioning PWT flow condition, are reported. 


\begin{tabular}{|c|c|}
\hline Chemical Specie & Highest concentration \\
\hline $\mathrm{N}_{2}$ & $7.8 \mathrm{E}-1$ \\
\hline $\mathrm{O}_{2}$ & $2.2 \mathrm{E}-1$ \\
\hline $\mathrm{N}$ & $4.5 \mathrm{E}-1$ \\
\hline $\mathrm{O}$ & $3.5 \mathrm{E}-1$ \\
\hline $\mathrm{Ar}$ & $2 \mathrm{E}-2$ \\
\hline $\mathrm{NO}$ & $2 \mathrm{E}-2$ \\
\hline $\mathrm{CO}_{2}$ & $1 \mathrm{E}-3$ \\
\hline $\mathrm{CO}$ & $1 \mathrm{E}-3$ \\
\hline $\mathrm{C}$ & $1 \mathrm{E}-3$ \\
\hline $\mathrm{NO}_{2}$ & $1 \mathrm{E}-5$ \\
\hline $\mathrm{CN}$ & $1 \mathrm{E}-5$ \\
\hline $\mathrm{N}_{2} \mathrm{O}$ & $1 \mathrm{E}-6$ \\
\hline $\mathrm{O}_{3}$ & $1 \mathrm{E}-7$ \\
\hline
\end{tabular}

Table 1: Computed highest concentration (molar fraction) by considering the whole operative running conditions in Scirocco Plasma Wind Tunnel

Our analysis about the behavior of such a plasma gas regarding emission and absorption of the radiation in the infrared region is performed by considering a gas mixture like the sum of the chemical species reported in Table 1 representing it the worst case. Our intention is to estimate the emission and absorption in IR spectrum in order to select a wavelength window where is possible to perform correctly the IR thermography measurements. We focus our analysis on the short-wave window $(2-6 \mu \mathrm{m})$ and long-wave window (8$14 \mu \mathrm{m})$, where commercial IR systems are available.

To perform the estimation of the spectral line intensity $\mathrm{S}$ of the chemical species reported in table 1, we used the HITRAN (acronym for highresolution transmission molecular absorption) database. Then, from these line intensity distributions and taking into account the thermodynamical conditions (temperature and partial pressure) and the maximum plasma thickness (about 1 meter), we compute the transmittance by using the ETRANS code by Ontar Co. In fig 1 the spectral line intensity of considered species are reported at $1000^{\circ} \mathrm{K}$ for the IR short-wave and IR long-wave windows, respectively. 


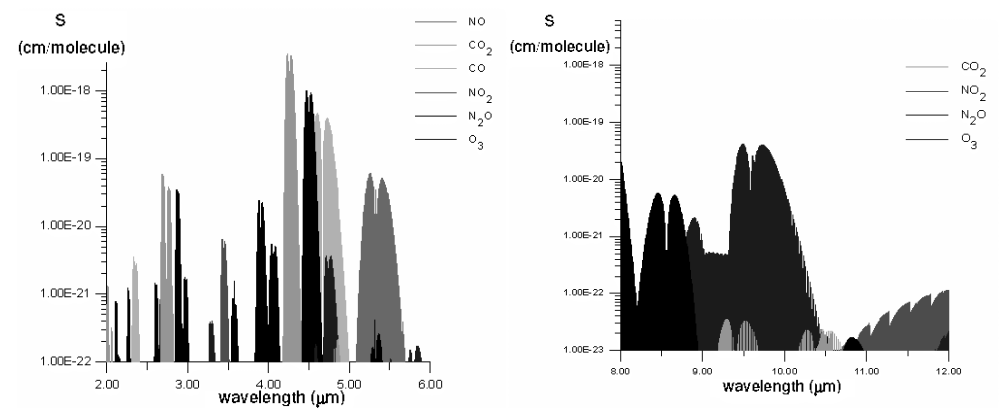

Figure 1: Spectral line intensity $S$ in the short-wave window

In fig. 2 the transmittance in the whole infrared region 1-12 $\mu \mathrm{m}$ is shown for two different temperatures of $300^{\circ} \mathrm{K}$ and $1000^{\circ} \mathrm{K}$, respectively.
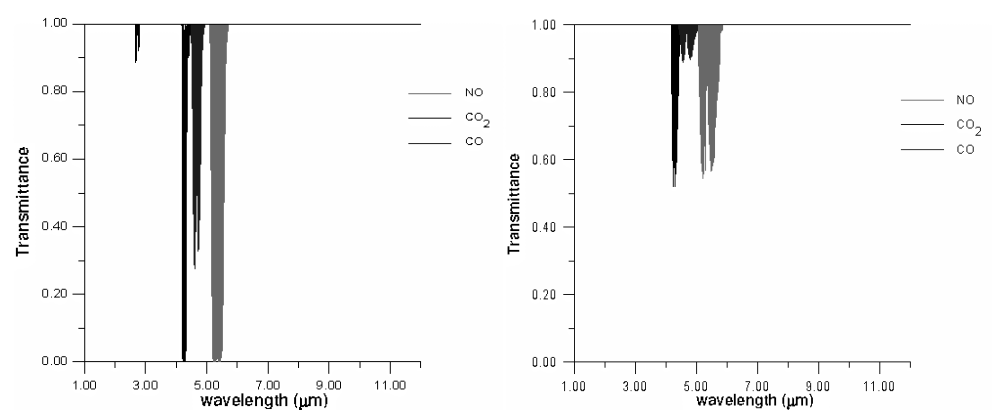

Figure 2: Transmittance at $300^{\circ} \mathrm{K}$ and $1000^{\circ} \mathrm{K}$

Following the results of this analysis a proper detector wavelength window and optics/filter have been determined in order to minimize the noise and any undesired source of disturbance. Then an application to a large scale test model (HYFLEX Nose Cap, overall dimension of about $80 \mathrm{~cm}$ ) was performed. The test campaign reproduced four flow conditions, two typical of the normal orbital reentry condition and other two based on the flight trajectory of Hyflex vehicle. In the table 2 all main flow parameters measured during the four test cases are reported. The test duration was fixed to around 30 seconds in order to preserve the heat shield material of the sample. Finally The wall heat flux are computed from the temperature time history under the assumption of semi infinite wall. 
http://dx.doi.org/10.21611/qirt.2004.091

\begin{tabular}{|c|c|c|c|c|c|c|c|}
\hline & $\begin{array}{c}\boldsymbol{M}_{\text {air }} \\
\boldsymbol{g} \boldsymbol{r} / \mathbf{s}\end{array}$ & $\begin{array}{c}\text { Power } \\
\boldsymbol{M W}\end{array}$ & $\begin{array}{c}\boldsymbol{H}_{\boldsymbol{0}} \\
\boldsymbol{M J} / \boldsymbol{K g}\end{array}$ & $\begin{array}{c}\boldsymbol{P}_{\boldsymbol{0}} \\
\boldsymbol{b a r}\end{array}$ & $\begin{array}{c}\boldsymbol{Q}_{\boldsymbol{s}} \\
\boldsymbol{K W} \mathbf{m}^{2}\end{array}$ & $\begin{array}{c}\boldsymbol{P}_{\boldsymbol{s}} \\
\boldsymbol{m b a r}\end{array}$ & $\begin{array}{c}\text { Time } \\
\text { Sec }\end{array}$ \\
\hline 1 & 520 & 11.3 & 11.9 & 2.45 & 590 & 7.6 & 32 \\
\hline 2 & 460 & 12.5 & 13.3 & 2.40 & 665 & 7.9 & 28 \\
\hline 3 & 1790 & 24.0 & 7.5 & 7.45 & 560 & 21.4 & 32 \\
\hline 4 & 1790 & 30.7 & 8.7 & 8.00 & 710 & 22.8 & 44 \\
\hline
\end{tabular}

Table 2: Main flow parameters in Hyflex test campaign. The stagnation heat flux $Q_{s}$ and pressure $P_{s}$ are the measured values onto the surface of the hemispherical $100 \mathrm{~mm}$ diameter cooled copper probe. 